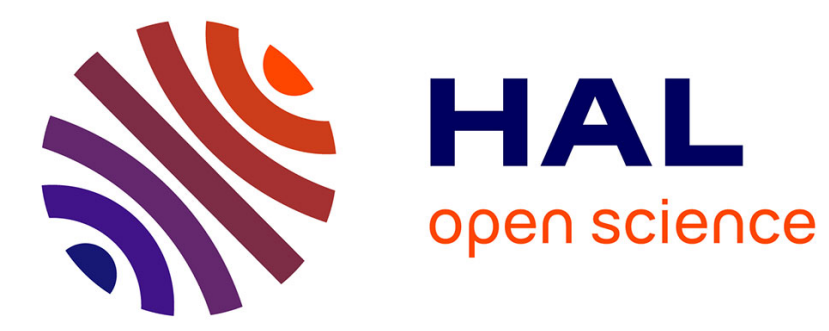

\title{
ELBOW POSITION SENSE IN MAN: CONTRASTING RESULTS IN MATCHING AND POINTING *
}

\author{
Jean-Luc Velay, Régine Roll, Jacques Paillard
}

\section{- To cite this version:}

Jean-Luc Velay, Régine Roll, Jacques Paillard. ELBOW POSITION SENSE IN MAN: CONTRASTING RESULTS IN MATCHING AND POINTING *. Human Movement Science, 1989, 8 (2), pp.177 - 193. 10.1016/0167-9457(89)90016-X . hal-01706487

\section{HAL Id: hal-01706487 \\ https://hal-amu.archives-ouvertes.fr/hal-01706487}

Submitted on 10 Apr 2018

HAL is a multi-disciplinary open access archive for the deposit and dissemination of scientific research documents, whether they are published or not. The documents may come from teaching and research institutions in France or abroad, or from public or private research centers.
L'archive ouverte pluridisciplinaire HAL, est destinée au dépôt et à la diffusion de documents scientifiques de niveau recherche, publiés ou non, émanant des établissements d'enseignement et de recherche français ou étrangers, des laboratoires publics ou privés. 


\title{
ELBOW POSITION SENSE IN MAN: CONTRASTING RESULTS IN MATCHING AND POINTING *
}

\author{
Jean-Luc VELAY, Régine ROLL and Jacques PAILLARD \\ Unité de Neurosciences Comportementales, Marseille, France
}

Velay, J.-L., R. Roll and J. Paillard, 1989. Elbow position sense in man: Contrasting results in matching and pointing. Human Movement Science 8, 177-193.

In this study an attempt is made to differentiate between two procedures which have been indiscriminately used in many previous studies to assess position sense in man: the matching procedure, in which subjects are required to place one arm in a position identical to that maintained by the contralateral target arm, and the pointing procedure, in which a given point on the target arm has to be touched with the index finger of the contralateral hand. For this purpose, eight subjects were tested in both tasks, before and after exposure of the target arm to $11 \mathrm{D}^{\circ}$ wedge prisms, on the assumption that matching and pointing performances would not be affected in the same way. The results show that this experimental manipulation affects only the pointing performances, indicating that matching and pointing tests involve separate central mechanisms for processing identical peripheral messages. It is suggested that, depending on the spatial requirements of the task, at least two types of coding of a given position might coexist at the central level.

Position sense can be broadly defined as the sense by which we can tell the position of our body parts when our eyes are closed. Such a general definition obviously lacks clarity when applied to notions like 'body schema', 'body image', 'kinaesthesia' or even 'proprioception'. The ambiguity of the concept has already been pointed out (Cohen 1958; McCloskey 1978; Paillard 1980) by authors attempting to evaluate whether the various experimental designs used in diverse disciplines ranging from neurology to neurophysiology and experimental psychology measure one and the same thing.

The classical procedure used to evaluate arm position sense is generally a 'matching procedure' in which an arm joint (target arm) is actively or passively moved, then stabilized at a given angle and the

* Requests for reprints should be sent to J.-L. Velay, Laboratoire de Neurobiologie Humaine, URA C.N.R.S. 372, Av. Escadrille Normandie Niemen, 13397 Marseille Cedex 13, France. 
subject is asked to match this posture with the contralateral arm (matching arm). Another way of assessing arm position sense is to use the 'pointing procedure' in which the subject has to reach with one arm (pointing arm) a target point on his other arm (target arm). A number of studies have been carried out in man using either matching (Goodwin et al. 1972; McCloskey 1973a, b; Soechting 1982; Soechting and Ross 1984; Worringham and Stelmach 1985; Worringham et al. 1987) or pointing tasks (Craske 1977; Craske and Crawshaw 1975; Paillard and Brouchon 1968, 1974; Slinger and Horsley 1906). The two procedures have sometimes been combined however, as when both reference and test movements are performed vertically, symmetrically about the sagittal plane. In this situation, both arms are held in the same posture with a close approximation between the two index fingers (Paillard and Brouchon 1968, 1974). Moreover, matching and pointing paradigms have sometimes led to divergent results. It has been reported, for example, that the time elapsing between positioning of target arm and performance of the pointing movement impairs the accuracy of pointing (Craske and Crawshaw 1975; Paillard and Brouchon 1968; Velay 1984), whereas with the matching procedure, increasing the interval between target arm positioning and matching caused no change in the accuracy of the performance (Horsh et al. 1975; Velay 1984). This difference suggests that the processes involved in memorizing the position might not be the same in both cases.

Although the need to distinguish between the representation of a limb posture and the location of a limb extremity has long been recognized (Paillard and Brouchon 1968), the question as to which spatial coordinate systems are used by the CNS to encode the positions of body parts has only recently been addressed (Soechting 1982; Soechting and Ross 1984; Worringham and Stelmach 1985; Worringham et al. 1987). Soechting (1982) and Soechting and Ross (1984) have proposed that elbow position sense may be described as the sense of orientation of the forearm in physical space. In a matching experiment designed to dissociate two coordinate systems, they observed that the subjects performed better when reproducing the forearm orientation using an 'absolute' frame of reference based on the sagittal and vertical planes than when matching a relative position such as the joint angle formed by the forearm in relation to the arm. Along similar lines, Worringham et al. (1987), while confirming these results, proposed additionally that proprioception be viewed as a system in which afferent 
signals relating to the gravitational torques acting on a joint lead to the perception of limb inclination rather than joint angles.

The following experiment was therefore designed to test the hypothesis that the central representation of a given position might differ depending on the test used to assess position sense. Matching and pointing procedures were compared under an unusual experimental condition intented to differentiate between them. For this purpose, the results of a single group of subjects were recorded in both tasks both before and after exposure of the target arm to 11 degrees wedge prisms. Indeed, the visuomotor recoordination which is observed after prismatic deviation of the visual field may offer a means of experimentally modifying the sense of position of a body segment. One of the many explanations advanced to account for prismatic adaptation has been that a proprioceptive change may occur in the felt position of the arm (Harris 1963) or, more specifically, in the transfer function of joint receptors (Craske 1966). In these authors' opinion, prismatic adaptation was clearly mediated by a change in position sense of the exposed arm. Efstathiou et al. (1967) and later Hardt et al. (1971) reported however that when, after prismatic exposure of one arm, instead of a pointing procedure to assess the recoordination process, the task consisted of reproducing with the same adapted arm a posture learned before exposure, no effects of prismatic exposure were observed in the subjects.

In our study, unlike the latter authors, we did not attempt to investigate the mechanisms involved and the place where adaptation occurs: prisms were used only to differentiate between the two procedures we were dealing with. In the classical methods used in prismatic adaptation studies, the prism-exposed arm is always used as active pointing arm during the post-exposure test, whereas here, it becomes the target. Furthermore, the limb which was used during the post-exposure task either to match the target arm posture or to point at the target hand, could never be seen by the subject during prism wearing, which was a major difference with previous studies.

We postulated that if the forearm joint position is really coded as a forearm orientation in space, then a shift will appear after prismatic exposure because prisms induce a general displacement of all the points along the body or the gaze axis. Conversely, if the forearm position is centrally represented as an angular position in relation to the upper arm, then no shift is to be expected after prismatic exposure. 
Elsewhere, in studies concerned with the central representation of limb position, only active positioning movements have been used. Now, the central coding of a position may differ depending on whether the positional signals are of peripheral and/or central origin. When the target arm is passively moved, the only available cues concerning the arm position originate from muscle, joint and skin receptors. It is reasonable to assume that the central command exerts no influence here. This is why two kinds of positioning movements (active and passive) were used in our experiments. We wanted to check whether the consequences of the prism adaptation of the target arm were identical when subjects assessed their arm position on the basis of peripheral information only, and when a contingent central component was added. It is conceivable, for instance, that an external reference frame might be used to centrally code a position when it is actively adopted, but not when it is imposed by the experimenter.

\section{Method}

\section{Subjects}

Eight right-handed students (four males and four females), who were naive as to the purpose of the experiment, participated as paid subjects in both the matching and pointing tests.

\section{Apparatus}

During the matching test, subjects sat comfortably in a chair with each arm on a support. The part supporting the forearm could be rotated without any friction around the axis coinciding with the elbow joint position (fig. 1a). The supports could be easily adjusted to the subjects' arm size, so that no resistance or discomfort prevented them from making forearm movements. The right arm was in a posture which was as similar as possible to that used in the pointing procedure (see fig. 1b) and, in particular the right index was maintained in an extended position, the index tip being in contact with the support. The angular positions of both right and left elbows were monitored by means of two high precision potentiometers (Helipot Beckman, linearity 


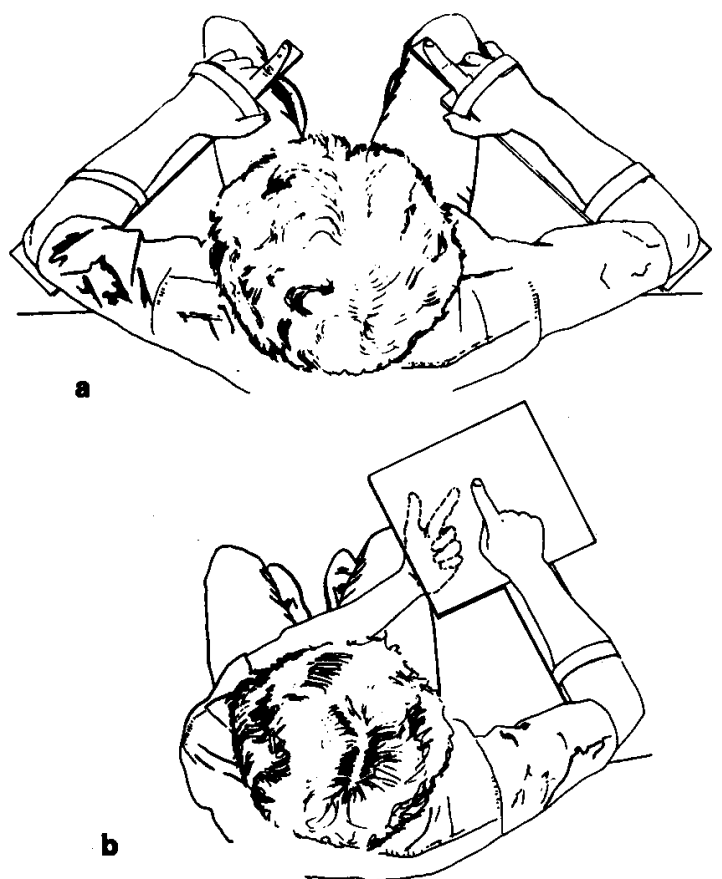

Fig. 1. Illustration of the experimental set-up: subject viewed from above in matching (a) and pointing (b) tasks.

$0.25 \%$ ) fixed beneath the rotation axes and stored on a computer after analog to digital conversion.

During the pointing test, the left support was removed. Furthermore, a grid-patterned printed circuit $(20 \mathrm{~cm} \times 20 \mathrm{~cm})$, the center of which coincided with the subject's index tip, was fixed beneath the right support (fig. 1b). The rectangular coordinates of the subject's left index finger position on the grid were recorded automatically as soon as he/she touched the grid. In both matching and pointing tasks, subjects wore goggles with removable shutter-flaps which were replaced during the exposure phase by $20 \mathrm{D}$ base right prisms producing a leftward binocular deviation of $11 \mathrm{deg}$.

\section{Procedure}

The aim of the experiment was to test position sense, in matching and pointing tasks, before and after prismatic exposure of the right 
arm. In both tests, the right arm was arbitrarily chosen as the 'target arm', the position of which had to be recognized; depending on the task, the left arm was used either as 'matching' or 'pointing' arm. Before testing, a training session was run so that the subjects could learn the three positions they had to reach and the mean movement speed they had to adopt under the active condition. The elbow flexion positions corresponded to angles of 50,70 and 90 degrees and the movement velocity was approximately $50 \mathrm{deg} / \mathrm{sec}$.

\section{Matching task}

The blindfolded subjects were seated with their two arms in the initial position, their elbows extended and forming an angle of $130 \mathrm{deg}$. Mechanical stops were fixed to the apparatus to ensure that the starting position remained constant throughout the experiment. The right (target) arm was then actively or passively flexed to a given position. When the movement was passive, the experimenter moved the subject's forearm up to the predetermined position. In the active situation, subjects were given a verbal command to move their arm towards one of the three angular positions they had previously learned. The end of the movement was automatically determined by the computer, which triggered a tone which was the command signal for left arm response. As soon as the left arm position was judged by the subjects to be correct, they informed the experimenter, who triggered the computer recording.

\section{Pointing task}

The pointing task procedure paralleled that of the matching task. The subjects' target arm was either actively or passively moved to a given position, while their pointing arm was placed on the left arm-rest. Then, when the tone occurred, they had to try to touch their right index with their left index. The initial touch only was considered and no corrective movement after the first touch was recorded.

\section{Prismatic exposure}

In both tests, the exposure conditions were identical. The blindfolded subjects were always seated in the experimental chair and a small table on which black targets were drawn was approached and 
placed in front of them. The right arm was placed on the table, whereas the left arm was not visible. The shutter-flaps were then replaced by the prisms and the subject was required to point with his right arm quickly towards the targets. The subject's head was free to move during exposure. After 5 minutes (sufficient time to allow total adaptation) and one hundred trials, the prisms were removed and replaced by shutter flaps. The subject's arms were replaced on the supports in preparation for the subsequent part of the experiment.

\section{Experimental design}

To avoid any undesirable bias introduced by prismatic exposure, a neutral exposure was used which was absolutely identical to the prismatic one except that neutral glasses were worn instead of prisms. Half of the subjects were then subjected to the experimental situations in the following order: neutral exposure - position sense test prismatic exposure - position sense test, and vice-versa for the other half: prismatic exposure - position sense test - neutral exposure - position sense test. Each experiment was divided into two sessions lasting approximately one hour each with a few minutes' rest interval. The first session was devoted to the matching task and the second to the pointing task. The three angular positions were used in both tests in a predetermined random sequence. Active and passive positioning was performed in blocks within which movements to each of the three positions occurred 5 times. Each subject performed 30 trials ( 5 trials $\mathrm{x} 3$ positions x 2 movement types) under both pre- and post-exposure conditions, i.e. 60 matching and 60 pointing trials.

\section{Data recording and analysis}

In the matching test, the subjects' performances were measured in terms of the difference between right and left elbow angular position. A positive value was assigned when the left arm was more flexed than the target one. The mean discrepancy between arm angles (constant error) and standard deviation (variability) were calculated in each experimental situation for each subject.

In the pointing task, the right index was placed in the center of the square grid on the origin of coordinate axes $(0,0)$. The accuracy of each pointing movement was measured in terms of two rectangular 


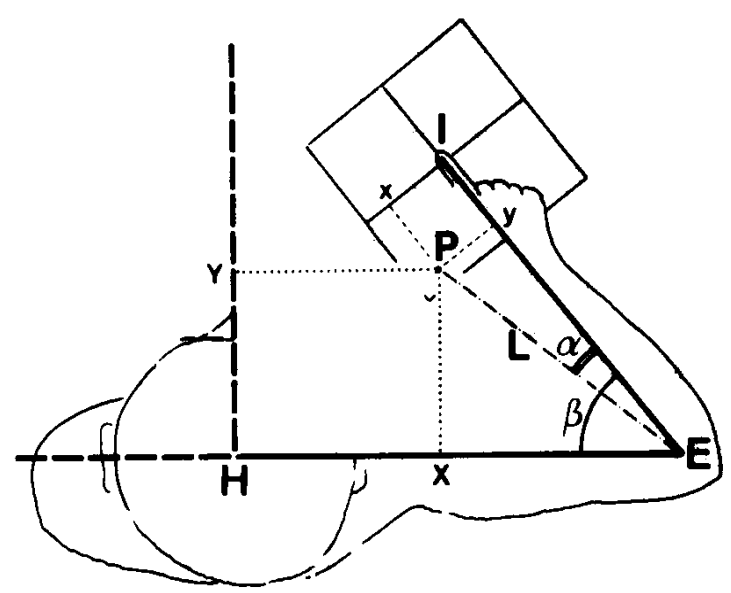

Fig. 2. Schematic representation of the various coordinate systems available for measuring pointing accuracy. /, $E$ and $H$ indicate the positions of the index tip, the elbow and the head center, respectively; $P$ denotes the final pointing position, $(x, y)$ are the coordinates of $P$ supplied by the electronic pointing grid; $(\alpha, L)$ are the angular elbow-centered coordinates calculated from $x$ and $y$; and $(X, Y)$ denote the rectangular coordinates of $P . \beta$ represents the measured angular position of the right support.

coordinates $(x, y)$ on the grid. Since the grid was fixed to the support, its orientation in space was dependent on the angular position of the elbow. To enable accuracy comparisons to be made over the whole range of positions, it was necessary to adopt a system of spatial coordinates which was independent of the forearm angular position. Fig. 2 shows several coordinate systems that can be used to determine the pointing error. One was the grid rectangular coordinates $(x, y)$ directly given by the display. Another could be derived from the transformation of the rectangular grid into elbow-centered polar coordinates $(\alpha, L) ; \alpha$ represents the angular error with respect to the forearm position and $L$ might be called the 'perceived' length of the limb (forearm, hand and finger). This coordinate system was appropriate for describing pointing accuracy because a was equivalent to the angular error measured in the matching test, and thus made comparisons possible between pointing and matching.

Furthermore, the index location might be defined in physical space by transforming the elbow centered polar coordinates $(\alpha, L)$ into rectangular coordinates $(X, Y)$ related to the external space. In fig. 2 , the coordinates have been centered on a point corresponding to the center of the head for computation convenience, but any other point could have been chosen. 
The anthropometric data used in the computations were measured at the beginning of the session, once the subject was seated in the apparatus.

The results of each task were subjected to a four-way analysis of variance ( 2 tasks x 2 exposures x 2 movement types x 3 positions).

\section{Results}

\section{Matching vs. pointing}

The overall results, in terms of the four experimental factors, are given in table 1 and plotted in fig. 3 . Errors in the pointing task refer to the elbow angular value $(\alpha)$ computed from the $(x, y)$ coordinates on the grid.

With both tasks and under most conditions, the constant error was positive, that is in the matching task the left forearm (matching forearm) was placed in a more flexed position than the right target arm and, in the pointing task, the pointing index fell left of the target index.

Table 1

Overall results in experiment 1 . $C E$ mean constant error, $S D$ : mean intra-individual variability.

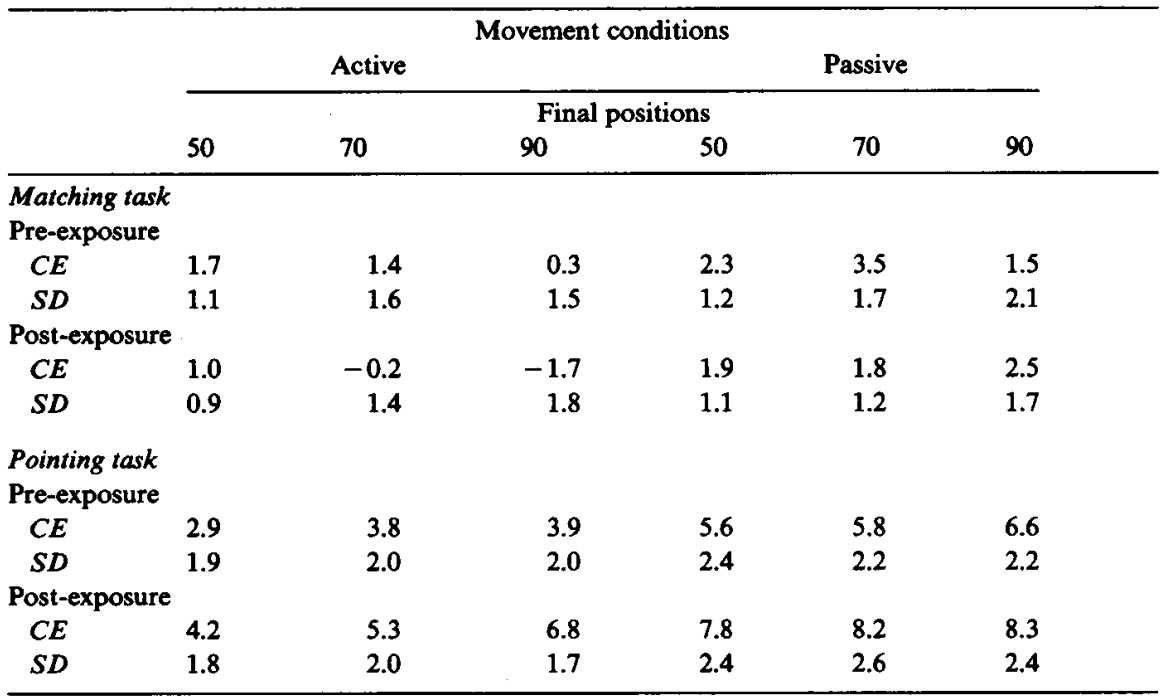



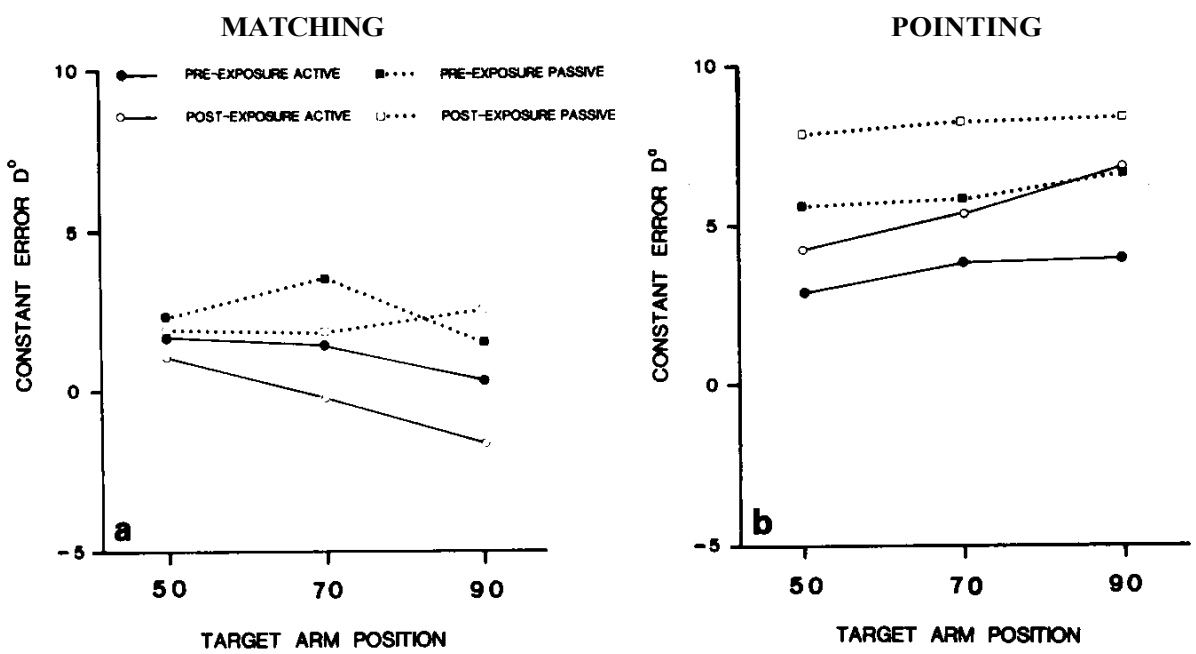

Fig. 3. Mean constant error in matching (a) and pointing (b) tasks.

In this task, errors were greater than those measured in matching $(F(1,7)=19.85, p<0.005)$.

Unlike the constant error, the intra-individual dispersion was less in the pointing than in the matching task $(F(1,9)=7.59, p<0.01)$.

\section{Final position}

In matching, in terms of constant error, no single angular position was better matched than the other two $(F(2,14)=1.86$, n.s. $)$. The intraindividual variability changed, however, as a function of the reference position; it was less pronounced when the position to be reproduced was more flexed $(F(2,14)=13.14, p<0.001)$. Each position gave rise to a different variability.

In pointing, the angular error decreased significantly when the right forearm was more flexed $(F(2,14)=13.83, p<0.001)$. Contrary to what was observed in the matching task, the intra-individual variability did not differ among the three positions.

Active vs. passive movement

In both tasks the constant error was significantly greater when the reference forearm movement was passively imposed than when it was actively performed $(F(1,7)=16.57, p<0.005)$. 
In matching the mean difference was $1.85 \mathrm{deg}, F(1,7)=7.07, p<$ $0.05)$. This difference was significant, however, only with positions at 70 and $90 \mathrm{deg}$.

In pointing, the mean constant error was 2.5 deg larger when the reference arm was passively positioned $(F(1,7)=36.54, p<0.001)$, in all three positions.

Generally speaking, no modification in the variability was observed whatever the type of movement performed. In pointing however, it was found to be greater after a passive movement, but this was significant only with the 70-deg position $(F(1,7)=11.61, p<0.05)$.

Pre-exposure vs. post-exposure

Both tasks combined, the differences observed between pre- and postexposure were below significance level $(F(1,7)=2.16$, n.s. $)$. However, as suggested in fig. 3 , the exposure seems to make the performances shift in opposite directions between the two tasks and in fact the cross-over interaction between the exposure and the task was significant $(F(1,7)=$ $14.53, p<0.01)$. However, the prism exposure appeared to significantly modify the performances only in the pointing (mean difference $=2 \mathrm{deg}$, $F(1,7)=22.17, p<0.005$ ) and not in the matching task (mean difference $=0.9 \mathrm{deg}, F(1,7)=2.13$, n.s.).

In matching, the difference between pre- and post-exposure was greater when the positioning was active $(d=1.43 \mathrm{deg})$ than when it was passive $(d=0.40 \mathrm{deg})$, but it was not significant $(F(1,7)=2.13$, n.s. $)$. Furthermore, the interaction between the prismatic exposure and the type of positioning movement was not significant $(F(1,7)<1)$.

In pointing, the difference had the same value whatever the mode of the target arm movement (1.9 deg with active and 2.2 deg for passive movements). In fact, the pointing area shifted leftwards after prismatic exposure and the change observed in the angular errors $(\alpha)$ was simply due to this shift. The right to left displacement of pointing becomes still more conspicuous if performance is described in terms of rectangular coordinates $(X, Y)$ referred to physical space (see fig. 2). In this case, only the lateral component $(X)$ was significantly modified and subjects perceived their index on average $1.8 \mathrm{~cm}$ more to the left than before exposure $(F(1,7)=16.28, p<0.01)$. The antero-posterior component $(Y)$ was not significantly influenced by prismatic exposure $(F(1,7)=$ 1.51, n.s.).

In both tasks, the variability was not affected by the prism exposure. 


\section{Discussion}

\section{Final position}

In both matching and pointing tests, the target forearm seemed to be generally perceived as being more flexed than it actually was, particularly when the position was passively adopted. This finding is comparable to the data reported in matching tasks involving the knee (Lloyd and Caldwell 1965) and the elbow (Worringham and Stelmach 1985). Likewise, the fact that the three target positions were not felt with equal accuracy is in agreement with Lloyd and Caldwell's reports (1965) that the extreme knee angles were more accurately perceived than the intermediate ones. In pointing experiments, it has also been reported that errors were such that the limb was apparently perceived as if it were closer to the body than it actually was (Gross et al. 1974; Slinger and Horsley 1906).

Active vs. passive movement

With both procedures, the fact that greater accuracy was obtained when the target position was adopted actively is in agreement with many studies on position sense involving a variety of different tasks (Craske and Crashaw 1975; Eklund 1972; Lloyd and Caldwell 1965; Paillard and Brouchon 1968, 1974).

In passive reaching a subject may evaluate his position only on the basis of peripheral signals originating from receptors located in joints, muscles and skin, whereas in active reaching, there exist several plausible possibilities. First, the content of peripheral signals is different in active movements. The discharge of joint receptors, for example, increases when muscular tension is applied to the joint capsule (Grigg 1975). Furthermore, the Golgi tendon organs, which are probably less involved during passive stretching or shortening of the muscle, can be activated when the muscle is voluntarily contracted (Rymer and D'Almeida 1980). On the other hand, although muscle spindle responses arising from stretched muscle are very similar whether the joint is rotated actively or passively (Roll and Vedel 1982), in the shortened agonist muscle, muscle spindle discharges, which are absent during passive shortening, greatly increase, at least during the isometric contraction corresponding to the holding of the final position. 
They might thus provide a signal which could effectively combine with information originating from the antagonist muscle. In this context, Paillard and Brouchon (1974) observed that when the agonist muscle was cooled or vibrated during active arm movement, the advantage of active positioning over passive positioning was lost. Finally, perception of position reached by an active movement might be based partly on information of central origin provided by the positioning commands which might be somehow combined with the peripheral signals.

\section{Pre-exposure vs. post-exposure}

The results show that the subjects' performances were not modified in the same way by prismatic exposure from one position sense test to another. This can be clearly seen from the interaction that existed between these two experimental factors. This interaction was due to the fact that the shift induced by the prismatic exposure was in opposite directions in the two tasks. Contrary to the pointing task, however, a shift was observed in only a few subjects in the matching task. One possible explanation for the opposite directions of the shift may be that these subjects referred the direction of their right forearm to an internal representation of their sagittal plane. If this straight ahead direction deviates towards the left after prism adaptation, the direction of the matching arm will then also deviate towards the left, which effectively corresponds to a smaller elbow angle error. In any case, the overall difference between pre- and post-exposure in the matching task was twice as low as in the pointing task and it did not reach significance level.

By contrast, all the subjects' performances were clearly modified in the pointing task after prismatic adaptation. It should be stressed that the observed pointing shift was due to the adaptation of the right target hand and that no such left-hand adaptation was observed because the subjects were always prevented from viewing their left arm during prismatic exposure. We cannot totally reject the possibility, however, that some intermanual transfer may have occurred, since the subjects' heads were not immobilized during exposure (Hamilton 1964; Wallace 1978); it is a well-known fact, nevertheless, that intermanual transfer also occurs under prismatic exposure conditions involving head restraint (Kalil and Freedman 1966). Finally, in subjects performing a task which consisted of attempting to touch a point on the left arm 
with the right hand, Craske (1966) observed a prism-induced error after the left hand had become adapted, although the subjects' head was immobilized. This result, which is along similar lines to ours, suggests that the shift observed in our experiment was not attributable to transfer. It may be on the contrary that, if any transfer occurred, it actually minimized the expected shift. In any case, if the pointing shift was due to a transfer of adaptation, it should have also occurred in the matching task, which was not the case.

The absence of any obvious shift in the matching task clearly rules out the purely proprioceptive hypothesis according to which prismatic adaptation is based entirely on a change in the angle signalled by the receptors (Craske 1966). Our results corroborate the conclusions reached by Efstathiou et al. (1967) and Hardt et al. (1971) according to whom prismatic adaptation occurs through a higher order modification.

Since the prismatic exposure conditions and the target arm positioning conditions were identical in both matching and pointing, we have come to the conclusion that these two tasks do not test the same position sense. By definition, a position can be specified only in relation to a reference; the question therefore arises as to what kind of reference system is involved in each task. Under our experimental conditions, the target arm movements were limited to the horizontal plane and one can assume that gravitational torques could not furnish any useful information about the arm position as suggested by Worringham et al. (1987). However, the forearm horizontal position could be coded in terms of a yaw angle relative to the sagittal plane as assumed by Soechting and Ross (1984). In that case one would expect to observe a shift in the matching task after prism exposure. When the position was actively attained, a little shift did in fact occur, the direction of which might suggest that there existed in a few subjects an inclination to adopt an external reference frame, such as the sagittal plane, in active positioning. However, using the sagittal plane did not seem to have been the most frequent strategy. Conversely, if the forearm position is not centrally represented as a forearm orientation in physical space, but rather as an angular position relative to the upper arm, it is not surprising that no shift was observed after prismatic exposure of the target arm.

The signals mediating position sensations, at least when the arm is passively displaced, are the proprioceptive messages generated by the 
diverse receptors located in the arm; now proprioceptors, whether they be articular, muscular or cutaneous, always discharge at a frequency which is closely related to the joint angle. We therefore suggest that the basic position sense is coded in terms of relative angular position in intrapersonal space. When no external reference is directly available, as in the horizontal matching task, the position can be more easily coded within this coordinate system.

If the CNS were endowed only with this type of angular representation ability, however, we would be unable to locate the extremities of our limbs in space. Another form of coding has to be envisaged that provides continuous knowledge of limb location in extrapersonal space. This other coding probably comes into play in the pointing task, which requires the target hand location to be determined. The CNS must then be somehow capable of performing the geometrical transformation from the postural space coordinates to the extrapersonal space coordinates.

We assume that the two types of position codings might coexist and correspond to two distinct, perhaps hierarchical, levels. To move from one level to the other, the CNS might use an extero-proprioceptive (mainly visuo-proprioceptive) relationship. This relationship is plastic thus providing a permanent adaptability to external changes. When it is artificially modified (by means of prisms for instance), the arm position in postural space is unchanged but new extrapersonal coordinates are associated with the angular coordinates and thus the postural configuration of the arm and the place of its extremity in physical space no longer coincide.

The distinction between those two levels is not purely speculative. Although parietal lesions often affect both types of activities, some patients have great difficulty in locating one of their hands (say the right hand) in space but are still able to reproduce with their left arm a posture passively imposed on their right arm. This type of disorder, in which hand location processes are damaged but postural reproduction processes preserved, is consistent with the possibility that the two position sense levels are hierarchically organized. This hierarchical model is incompatible, however, with the existence of a lower level selective impairment and if it is valid, it will be probably impossible to find patients who are unable to perform a matching task but capable of locating their hand correctly in space. Now, Norsell (1970) and McCloskey (1973b) have described a patient with a rather complex cerebral 
lesion which made him totally unable to reproduce with one arm a posture passively imposed on the contralateral arm. Strangely enough, this man did not seem to be at all handicapped in spatial activities requiring localization of his hands without visual control. If other such patients are found to exist, a strict hierarchy hypothesis will have to be rejected in favour of a two independent parallel level model, the one level coding joint angle and the other specifying hand location.

The present experimental data suggest that the coordinate representation of arm position might be manifold and that the choice of experimental procedure used to assess subjects' position sense could be crucial. In an initial approach, a matching task involving the processing of proprioceptive messages in postural space seems to be more appropriate for investigating joint position sense, that is positions coded in terms of relative configurations. If any external reference (such as the gravitational vertical reference) is available, however, it will probably be advantageous for the CNS to use a position representation system based on an extrapersonal frame of reference, even in a matching procedure. This second order coding may be automatically called up in pointing tasks because here position is treated like location in extrapersonal space.

\section{References}

Cohen, L.A., 1958. Analysis of position sense in human shoulder. Journal of Neurophysiology 21,550-562.

Craske, B., 1966. Change in transfer function of joint receptor output. Nature 210, 764765.

Craske, B., 1977. Perception of impossible limb positions induced by tendon vibration. Science 196, 71-73.

Craske, B. and Crawshaw M., 1975. Shifts in kinesthesis through time and after active and passive movement. Perceptual and Motor Skills 40, 755-761.

Efstathiou, A., J. Bauer, M. Greene and R. Held, 1967. Altered reaching following adaptation to optical displacement of the hand. Journal of Experimental Psychology $73,113-120$.

Eklund, G., 1972. Position sense and state of contraction; the effects of vibration. Journal of Neurology, Neurosurgery and Psychiatry 35, 606-611.

Goodwin, G.M., D.I. Mc Closkey and P.B.C. Matthews, 1972. The contribution of muscle afferents to kinaesthesia shown by vibration induced illusions of movement and by effects of paralysing joint afferents. Brain 95, 705-748.

Grigg, P., 1975. Mechanical factors influencing response of joint afferent neurons from cat knee. Journal of Neurophysiology 38, 1473-1484.

Gross, Y., R. Webb and R. Melzack, 1974. Central and peripheral contributions to localization of body parts: Evidence for a central body schema. Experimental Neurology 44, 346-362.

Hamilton, C.R., 1964. Intermanual transfer of adaptation to prisms. American Journal of Psychology 77, 457-462. 
Hardt, M.E., R. Held and M.J. Steinbach, 1971. Adaptation to displaced vision: a change in the central control of sensorimotor coordination. Journal of Experimental Psychology 89, 229-239.

Harris, C.S., 1963. Adaptation to displaced vision: visual, motor or proprioceptive change? Science 140, 812-813.

Horsh, K.W., F.J. Clark and P.R. Burgess, 1975. Awareness of knee joint angle under static conditions. Journal of Neurophysiology 38, 1436-1447.

Kalil, R.E. and S.J. Freedman, 1966. Intermanual transfer of compensation for displaced vision. Perceptual and Motor Skills 22, 123-126.

Lloyd, A. and L. Caldwell, 1965. Accuracy of active and passive positioning of the leg on the basis of kinesthetic cues. Journal of Comparative and Physiological Psychology 60, 102-106.

McCloskey, D.I., 1973a. Differences between the senses of movement and position shown by the effects of loading and vibration of muscle in man. Brain Research 63, 119-131.

McCloskey, D.I., 1973b. Position sense after surgical disconnexion of the cerebral hemispheres in man. Brain 96, part II, 269-276.

McCloskey, D.I., 1978. Kinaesthetic sensibility. Physiological Reviews 58, 763-820.

Norsell, V., 1970. Some aspects of proprioception after forebrain commissurotomy in man. Acta Physiologica Scandinavia 79, 35A-37A.

Paillard, J., 1980. Le corps situé et le corps identifié: une approche psychophysiologique de la notion de schéma corporel. Revue Médicale de la Suisse Romande $100,129-141$.

Paillard, J. and M. Brouchon, 1968. 'Active and passive movements in the calibration of position sense'. In: S.J. Freedman (ed.), The neuropsychology of spatially oriented behavior. Homewood, IL: Dorsey Press, pp. 37-55.

Paillard, J. and M. Brouchon, 1974. A proprioceptive contribution to the spatial encoding of position cues for ballistic movements. Brain Research 71, 273-284.

Roll, J.P. and J.P. Vedel, 1982. Kinaesthetic role of muscle afferents in man, studied by tendon vibration and microneurography. Experimental Brain Research 47, 177-190.

Rymer, W.Z. and A. D'Almeida, 1980. Joint position sense: the effects of muscle contraction. Brain 103, 1-22.

Slinger, R.T. and V. Horsley, 1906. Upon the orientation of points in space by muscular, arthroidal and tactile senses of the upper limbs in normal individuals and in blind persons. Brain 29, 1-27.

Soechting, J.F., 1982. Does position sense at the elbow reflect a sense of elbow joint angle or one of limb orientation? Brain Research 248, 392-395.

Soechting, J.F. and B. Ross, 1984. Psychophysical determination of coordinate representation of human arm orientation. Neuroscience 13, 595-604.

Velay, J.L., 1984. Sens de la position: Les traitements sensori-moteurs des informations proprioceptives. Thèse de Doctorat de III cycle, Université d'Aix-Marseille II.

Wallace, B., 1978. Visuomotor coordination and intermanual transfer for a proprioceptive reaching task. Journal of Motor Behavior 10, 139-147.

Worringham, C.J. and G.E. Stelmach, 1985. The contribution of gravitational torques to limb position sense. Experimental Brain Research 611, 38-42.

Worringham, C.J., G.E. Stelmach and Z.E. Martin, 1987. Limb segment inclination sense in proprioception. Experimental Brain Research 66, 653-658. 